\title{
Model of Advancing and Receding Contact Angles on Rough Surfaces
}

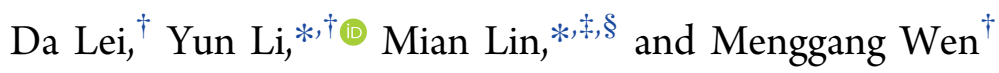 \\ ${ }^{\dagger}$ School of Chemical Engineering and Technology, Xi’an Jiaotong University, Xi’an, Shaanxi 710049, China \\ ${ }^{\ddagger}$ Institute of Mechanics, Chinese Academy of Sciences, Beijing 100190, China \\ ${ }^{\S}$ School of Engineering Science, University of Chinese Academy of Sciences, Beijing 100049, China
}

\section{Supporting Information}

\begin{abstract}
Contact angle hysteresis is a basic phenomenon in many industrial applications, such as material surface engineering and enhanced oil recovery. The mechanism of continuous change of the contact angle during hysteresis is not well understood and described, and it is also important to predict advancing and receding angles by the Young contact angle and surface roughness. In this work, a contact angle hysteresis model is developed based on several metastable contact angle models. The fraction difference between gas and liquid prefilling the solid grooves is assumed to be variable, so is the fraction difference of gas and liquid displaced by the other phase. Besides these two

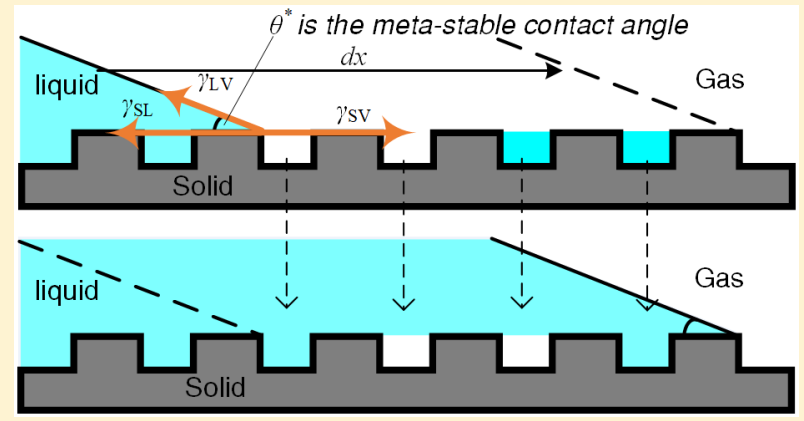
newly introduced variables, the solid area fraction, the ratio of the real surface area to the projected surface area, and the Young contact angle together influence the advancing and receding angles. Energy input or work is the external cause of hysteresis. The new variables are the intrinsic cause of hysteresis, enabling the continuous change of the contact angle and ensuring a minimal surface energy during hysteresis. The advancing and receding angles are explicitly expressed in a function of the factors mentioned above. The results from this model are in good coincidence with experiments from the literature. Through comparison, it is found that the model in this paper can be reduced as the Wenzel model, Cassie-Baxter model, etc., under special conditions.
\end{abstract}

\section{INTRODUCTION}

The Young contact angle is an important parameter characterizing the wettability of a solid-liquid-liquid or solid-liquidgas system. Based on Young's theory, the apparent contact angle represents the balance of interfacial tensions. ${ }^{1}$ However, experiments have found that the contact angle may vary within a certain range with contact points remaining static, meaning $\theta_{\mathrm{o}}$ $\in\left[\begin{array}{ll}\theta_{\mathrm{adv}}, & \theta_{\mathrm{rec}}\end{array}\right]$, wherein $\theta_{\mathrm{adv}}$ and $\theta_{\mathrm{rec}}$ are the advancing and receding threshold angle, respectively. ${ }^{2}$ The difference between the advancing and receding angle is defined as the contact angle hysteresis

$$
\Delta \theta_{\text {hys }}=\theta_{\text {adv }}-\theta_{\text {rec }}
$$

Contact angle hysteresis is closely related to industrial applications, such as surface self-cleaning, ${ }^{3}$ mobilization of bubbles in oil reservoirs during oil extraction, ${ }^{4}$ etc. A better understanding of contact angle hysteresis facilitates the control of the wettability of solid materials, which is the main interest and key issue in surface engineering. ${ }^{5}$

Surface roughness and chemical and topographical heterogeneities are recognized as the main causes of contact angle hysteresis. ${ }^{2}$ The lotus leaves are known to repel a water droplet due to their surface structures, with the advancing angle of $160^{\circ}$ and the receding angle of $150^{\circ} .5$ The hydrophobic microscale hairs on the legs allow the microvelia to walk on water. ${ }^{6}$ Jamin first observed that a series of bubbles in a capillary can withstand a certain amount of pressure without moving, ${ }^{7}$ which was found to be caused by contact angle hysteresis. ${ }^{8}$ Smith and Crane found that a capillary contaminated by a solution of oleic acid in benzene demonstrate larger hysteresis than clean capillary. Morrow et al. measured the advancing and receding angles of different liquids in capillaries with roughened and unroughened inner surfaces and found that the hysteresis in the roughened capillary is more obvious than in the unroughened one. ${ }^{9}$ Meiron et al. measured the advancing and receding angles of water and ethylene glycol on abrasive papers coated with beeswax, and the contact angle hysteresis was found to increase as the increase of the arithmetical mean deviation of the vertical profile of abrasive papers. ${ }^{10}$ Experimental results also showed that the density of surface defect can affect the contact angle hysteresis: a hysteresis energy defined as $\gamma\left(\cos \theta_{\text {rec }}-\cos \theta_{\text {adv }}\right)$ is proportional to the density of surface defect when defects are dilute distributed, wherein $\gamma$ is the surface tension; when the defects are densely distributed, the hysteresis energy decrease as the defect density increases. $^{11,12}$

Received: April 9, 2019

Revised: July 4, 2019

Published: July 5, 2019 


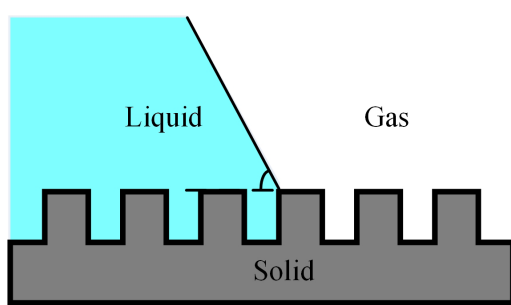

(a)Wenzel state

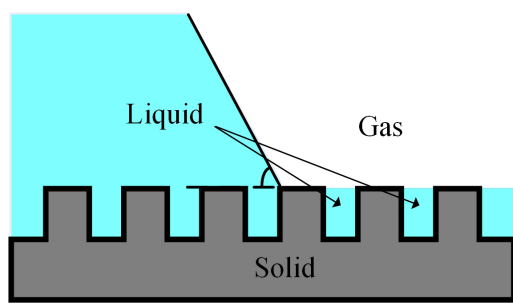

(c)Impregnating state

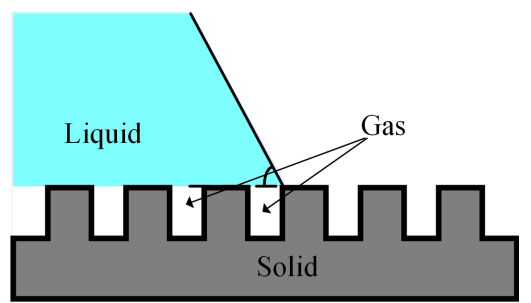

(b)Cassie-Baxter state

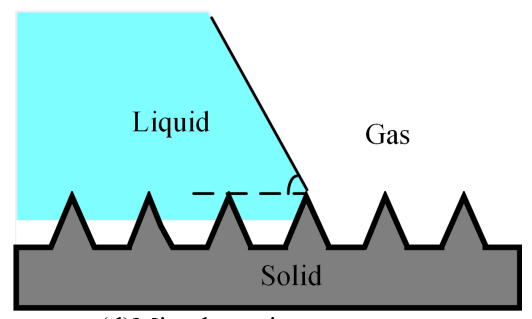

(d)Mixed-wetting state

Figure 1. Different metastable wetting state.

For rough surfaces, the advancing and receding contact angles are the most essential parameters. It is very important to understand how these angles can be calculated from the Young angle. The quantitative relationship between the contact angle hysteresis and the surface roughness also remains to be fully understood. ${ }^{5}$ Young's model assumes that the solid surface is perfectly smooth, the apparent contact angle can be expressed by interfacial tensions (subscripts $\mathrm{S}, \mathrm{V}$, and $\mathrm{L}$ denotes solid, vapor, and liquid, respectively)

$$
\cos \theta_{\mathrm{o}}=\frac{\gamma_{\mathrm{SV}}-\gamma_{\mathrm{SL}}}{\gamma_{\mathrm{LV}}}
$$

1.1. Metastable Contact Angle. Wenzel considered the situation of the rough solid surface and assumed that the liquid enters the space of surface defects, as depicted in Figure $1 \mathrm{a} .{ }^{13}$ To achieve the minimum energy variation in Wenzel's conditions, the contact angle $\theta_{\mathrm{W}}$ may vary from the Young contact angle as follows

$$
\cos \theta_{\mathrm{W}}=r \cos \theta_{\mathrm{o}}
$$

The roughness factor $r$ is defined as the ratio of the real surface area to its horizontally projected area. Cassie and Baxter proposed an opposite model, which assumed that air remains in the surface defects and forms air pockets beneath the liquid as depicted in Figure 1b. ${ }^{14}$ The apparent contact angle under the state of Cassie-Baxter is given by eq 4 , wherein $\phi_{\mathrm{S}}$ represents the area fraction, where the liquid contacts the solid.

$$
\cos \theta_{\mathrm{CB}}=\phi_{\mathrm{S}}\left(\cos \theta_{\mathrm{o}}+1\right)-1
$$

Despite some experimental results are in good coincidence with the Wenzel model, ${ }^{10}$ Wolansky and Marmur provided mathematical proofs that the Wenzel model only applies to the case where the size of the liquid drop is much larger than the surface roughness. ${ }^{15}$ Furthermore, the applicability of the Wenzel model and the Cassie-Baxter model is still controversial. $^{2}$

Researchers further developed the Cassie-Baxter model into an impregnating model, as depicted in Figure 1c. ${ }^{5,16,17}$ In this model, the liquid penetrates into the surface defects under the conditions of $\cos \theta_{\mathrm{o}}>\left(1-\phi_{\mathrm{S}}\right) /\left(r-\phi_{\mathrm{S}}\right)$, and the apparent contact angle $\theta_{\mathrm{im}}$ is given by

$$
\cos \theta_{\text {im }}=\phi_{\mathrm{S}}\left(\cos \theta_{\mathrm{o}}-1\right)+1
$$

However, a pure state of Wenzel or Cassie-Baxter is rare in real situations. ${ }^{18}$ A mixed-wetting state is proposed, which assumed partial penetrations of the liquid into the solid grooves, as depicted in Figure 1d. ${ }^{19-21}$ The fraction of the projected area wetted by the liquid is denoted by $\phi_{S}^{\prime}$, and the apparent contact angle $\phi_{\text {mix }}$ is given by eq 6 . When $\phi_{S}^{\prime}=1$, the mixed model eq 6 can be reduced as the Wenzel model eq 3 .

$$
\cos \theta_{\text {mix }}=\phi^{\prime}{ }_{S}\left(r \cos \theta_{\mathrm{o}}+1\right)-1
$$

1.2. Hysteresis Energy. Joanny and de Gennes provided another approach to describe the contact angle hysteresis. ${ }^{22}$ They derived an analytical solution for the energy dissipated during the contact line getting over a single surface defect $W_{\mathrm{d}}$.

$$
W_{\mathrm{d}} \sim \frac{\delta S^{2} d^{2}}{\gamma_{\mathrm{LV}} \sin ^{2} \theta_{\mathrm{o}}}
$$

where $d$ represents the characteristic size of surface defects and $\delta S$ is the random fluctuation of a spreading coefficient, which is defined as ${ }^{2}$

$$
\delta S=\gamma_{\mathrm{LV}}\left(\cos \theta-\cos \theta_{\mathrm{o}}\right)
$$

where $\theta$ characterizes the contact angle when the contact line mounts on a surface defect. The total change of energy during hysteresis consists of the energy dissipated in the advancing and receding direction, over several surface defects. The hysteresis increases linearly with the number of sparsely distributed defects and it gives ${ }^{2}$

$$
\gamma_{\mathrm{LV}}\left(\cos \theta_{\text {rec }}-\cos \theta_{\mathrm{adv}}\right) \sim n \frac{\delta S^{2} d^{2}}{\gamma_{\mathrm{LV}} \sin \theta_{\mathrm{o}}}
$$

However, the hysteresis model proposed by Joanny and de Gennes is limited to mesoscopic defects. ${ }^{2}$ Koch et al. developed a hysteresis model based on the theory of Joanny and de Gennes, and the Cassie-Baxter model. ${ }^{23}$ Advancing and receding pinning forces $F_{\mathrm{P}, \text { adv }} / F_{\mathrm{P} \text {,rec }}$ are assumed to cause the difference between $\theta_{\mathrm{adv}} / \theta_{\mathrm{rec}}$ and $\theta_{\mathrm{CB}}$, as given in eq 10 , which further yields the function of $\theta_{\mathrm{o}}$. 


$$
\begin{aligned}
& \left\{\begin{array}{l}
\cos \theta_{\mathrm{adv}}=\cos \theta_{\mathrm{CB}}-\frac{F_{\mathrm{P}, \mathrm{adv}}}{\gamma_{\mathrm{LV}}} \\
\cos \theta_{\mathrm{rec}}=\cos \theta_{\mathrm{CB}}+\frac{F_{\mathrm{P}, \mathrm{rec}}}{\gamma_{\mathrm{LV}}}
\end{array}\right. \\
& \Rightarrow\left\{\begin{array}{l}
\cos \theta_{\mathrm{adv}}=\phi_{\mathrm{S}}\left(\cos \theta_{\mathrm{o}}+1\right)-1-\frac{F_{\mathrm{P}, \mathrm{adv}}}{\gamma_{\mathrm{LV}}} \\
\cos \theta_{\mathrm{rec}}=\phi_{\mathrm{S}}\left(\cos \theta_{\mathrm{o}}+1\right)-1+\frac{F_{\mathrm{P}, \mathrm{rec}}}{\gamma_{\mathrm{LV}}}
\end{array}\right.
\end{aligned}
$$

In eq $10, F_{\mathrm{P}, \text { adv }}$ and $F_{\mathrm{P} \text {,rec }}$ are unknown parameters and need to be fitted by experiments. However, in comparison with experiments, $F_{\mathrm{P}, \mathrm{adv}}$ was treated as a linear function of $\phi_{\mathrm{S}}$ and an additional parameter was added to the model. Moreover, the physical meaning of $F_{\mathrm{P}, \text { adv }}$ or $F_{\mathrm{P} \text {,rec }}$ is still blurred and this model needs further development.

1.3. Empirical Relationship. Based on experimental observations, Lei et al. proposed a linear relationship between the advancing angle $\theta_{\mathrm{adv}}$ and the receding angle $\theta_{\text {rec }}{ }^{24}$

$$
\theta_{\mathrm{rec}}=k \theta_{\mathrm{adv}}
$$

wherein $k$ is the linear factor and it is found to be positively correlated with the surface roughness (root mean square roughness or average profile deviation). Despite numbers of experiments are in good coincidence with eq 11, the linear relationship between $\theta_{\text {adv }}$ and $\theta_{\text {rec }}$ lacks a theoretical basis and needs more verification.

In summary, metastable contact angle models, such as the Wenzel model, the Cassie-Baxter model, and the impregnating model, explain how the contact angle may vary under different situations to satisfy the principle of minimizing the surface energy. These models cannot explain the existence of multiple metastable contact angles during hysteresis. ${ }^{15}$ The hysteresis model proposed by Joanny and de Gennes cannot be applied to situations where the surface roughness is nanoscale. ${ }^{2}$ The mixedwetting model and the model proposed by Koch have the potential to tackle the unexplained problems, but further verification needs to be done. Empirical models should be compared with theoretical models to gain a theoretical basis. Although metastable contact angles only present lowest surface energy under given states and do not directly relate to the contact angle hysteresis, hysteresis can be accounted for if there is a way to describe the transition between different metastable contact angles or wetting states. The aim of this work is to develop a hysteresis model based on the analysis of surface energy as in other metastable contact angle models so that the relationship between $\theta_{\text {adv }} / \theta_{\text {rec }}$ and $\theta_{\mathrm{o}}$ can be obtained.

\section{THEORY}

2.1. Assumptions and Equilibrium Analysis. It is assumed that the solid surface is chemically homogeneous, and defects (grooves or posts) are uniformly distributed. The length and height of a post are denoted $L_{\mathrm{d}}$ and $h_{\mathrm{d}}$, respectively, and the width of a groove is denoted $w_{\mathrm{d}}$. Unlike the Wenzel, the Cassie-Baxter and the impregnating model, it is further assumed that the surface defects cannot be fully occupied by either liquid or gas. The fraction of liquid or gas penetrating grooves is now variable, as depicted in Figure 2. According to its original definition, the fraction of the wetted area without penetration $\phi_{\mathrm{S}}$ can be calculated as in eq 12

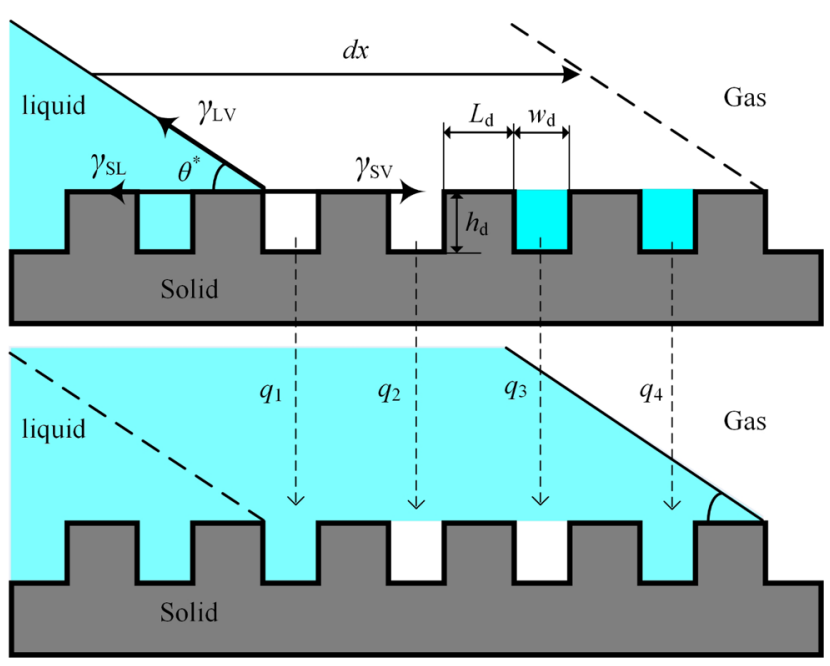

Figure 2. Surface topography, the fraction of gas and liquid prefilling the grooves and the fraction of gas and liquid displaced by the other phase, together influence the quasi-static contact angle during the contact point moving forward for a distance of $\mathrm{d} x$.

$$
\phi_{\mathrm{S}}=\frac{L_{\mathrm{d}}}{L_{\mathrm{d}}+w_{\mathrm{d}}}
$$

Parameter $r$ denotes the ratio of the real area of the rough surface to its projected area, according to the Wenzel model, which is given by

$$
r=\frac{2 h_{\mathrm{d}}}{L_{\mathrm{d}}+w_{\mathrm{d}}}+1
$$

Let us assume that the three-phase point moves forward (liquid displaces gas) and cover a range of $\mathrm{d} x$ under quasi-static conditions. As demonstrated in Figure 2, interfaces may form or vanish during the movement, which includes

1. Solid-gas interfaces turn into solid-liquid interfaces on the top of defects with the fraction of $\phi_{S}$. The change in surface energy is $\phi_{\mathrm{S}}\left(\gamma_{\mathrm{SL}}-\gamma_{\mathrm{SV}}\right) \mathrm{d} x$.

2. The fraction of the gas and liquid in grooves are $\phi_{\mathrm{V}}$ and $\phi_{\mathrm{L}}$, respectively, which satisfies $1-\phi_{\mathrm{S}}=\phi_{\mathrm{V}}+\phi_{\mathrm{L}}$. During the movement, grooves originally occupied by gas will be penetrated by the liquid, with the quantity of $q_{1}$. Solidgas interfaces will be displaced by solid-liquid interfaces, and the change of surface energy is $q_{1}\left(1+2 h_{\mathrm{d}} / w_{\mathrm{d}}\right)\left(\gamma_{\mathrm{SL}}-\right.$ $\left.\gamma_{\mathrm{SV}}\right) \mathrm{d} x$; the fraction $q_{2}$ of gas-occupied grooves will remain unchanged and the new liquid-gas interfaces will form upon the grooves, the change in surface energy being $q_{2} \gamma_{\mathrm{LV}} \mathrm{d} x$.

3. Grooves originally occupied by liquid will be penetrated by gas, with the quantity of $q_{3}$. Solid-liquid interfaces will be displaced by solid-gas interfaces, and the change of surface energy is given by $-q_{3}\left(1+2 h_{\mathrm{d}} / w_{\mathrm{d}}\right)\left(\gamma_{\mathrm{SL}}-\gamma_{\mathrm{SV}}\right) \mathrm{d} x$; while the other part $q_{4}$ of liquid-occupied grooves will remain unchanged, but previous liquid-gas interfaces will vanish, the change of surface energy being $-q_{4} \gamma_{\mathrm{LV}} \mathrm{d} x$.

4. The liquid-gas interfacial tension $\gamma_{\mathrm{LV}}$ does work along the distance $\mathrm{d} x$, increasing the surface energy by $\gamma_{\mathrm{LV}} \cos \theta^{*}$ $\mathrm{d} x$.

The total change in surface energy $d E$ is given by the sum of the aforementioned parts 


$$
\begin{aligned}
\mathrm{d} E= & {\left[\phi_{\mathrm{S}}+\left(q_{1}-q_{3}\right) \frac{2 h_{\mathrm{d}}+w_{\mathrm{d}}}{w_{\mathrm{d}}}\right]\left(\gamma_{\mathrm{SL}}-\gamma_{\mathrm{SV}}\right) \mathrm{d} x } \\
& +\left(q_{2}-q_{4}\right) \gamma_{\mathrm{LV}} \mathrm{d} x+\gamma_{\mathrm{LV}} \cos \theta^{*} \mathrm{~d} x
\end{aligned}
$$

where the fraction $q_{1}$ and $q_{2}$ make up the total fraction of grooves initially occupied by gas, $q_{1}+q_{2}=\phi_{\mathrm{V}}$; the fraction $q_{3}$ and $q_{4}$ make up the total fraction of grooves initially occupied by the liquid, $q_{3}$ $+q_{4}=\phi_{\mathrm{L}}$. The three-phase system reaches equilibrium when $\mathrm{d} E$ is minimal (zero). ${ }^{5,16,17}$ Combining eqs $2,12,13$, and $14, \mathrm{~d} E=0$ yields

$$
\cos \theta^{*}=\left[\phi_{\mathrm{S}}+\left(q_{1}-q_{3}\right) \frac{r-\phi_{\mathrm{S}}}{1-\phi_{\mathrm{S}}}\right] \cos \theta_{\mathrm{o}}-\left(q_{2}-q_{4}\right)
$$

Now, the expression of the quasi-static contact angle is obtained. The scope and physical meaning of $q_{1}, q_{2}, q_{3}$, and $q_{4}$ will be discussed below, in comparison with other metastable contact angle models.

2.2. Comparison with Metastable Contact Angle Models. It has been recognized that the apparent contact angle may continuously change, or there are multiple metastable contact angles, during hysteresis. ${ }^{15,25}$ As for two-phase displacement in capillaries, the pressure difference along the capillary can be changed to induce the contact angle hysteresis. ${ }^{4}$ To observe hysteresis with the sessile drop method, the plate holding the droplet can be tilted and the droplet will deform due to gravitation. ${ }^{26}$ Vibrating the solid surface can also influence the droplet contact angle. ${ }^{10}$ In all, external energy input or work brings change to the surface energy. To maintain equilibrium, the contact angle has to vary.

The variability of $q_{1}, q_{2}, q_{3}$, and $q_{4}$ provide a way to explain the intrinsic mechanism of how the three-phase system adapts to external disturbance and maintains equilibrium. According to the above discussion, $q_{1}, q_{2}, q_{3}$, and $q_{4}$ are non-negative parameters, and they satisfy $q_{1}+q_{2}+q_{3}+q_{4}=1-\phi_{\mathrm{S}}$. For the convenience of discussion, $q_{1}-q_{3}$ and $q_{2}-q_{4}$ are replaced by $t_{1}$ and $t_{2}$, respectively, in eq 15 , which yields

$$
\cos \theta^{*}=\left(\phi_{\mathrm{S}}+t_{1} \frac{r-\phi_{\mathrm{S}}}{1-\phi_{\mathrm{S}}}\right) \cos \theta_{\mathrm{o}}-t_{2}
$$

By their definitions, $t_{1}$ and $t_{2}$ satisfy $\left|t_{1}\right| \leq 1-\phi_{S},\left|t_{2}\right| \leq 1-\phi_{S}$, $\mid t_{1}$ $+t_{2} \mid \leq 1-\phi_{S}$, and $\left|t_{1}-t_{2}\right| \leq 1-\phi_{S}$. $t_{1}$ represents the difficulty (probability) of liquid displacing gas in the grooves; $t_{2}$ represents the tendency (probability) of liquid or gas remaining in the grooves. $t_{1}$ and $t_{2}$ may vary according to the external energy input. Their variability enables the continuous change of the contact angle and ensures a minimal surface energy $(\mathrm{d} E=0)$ during hysteresis.

If all grooves are preoccupied by gas and are not to be penetrated by liquid, i.e., $t_{1}=0$ and $t_{2}=1-\phi_{\mathrm{S}}\left(q_{2}=1-\phi_{\mathrm{S}}\right.$ and $q_{1}=q_{3}=q_{4}=0$ in Figure 2), eq 16 turns into $\cos \theta^{*}=\phi_{\mathrm{S}} \cos \theta_{\mathrm{o}}-$ $1+\phi_{S}$, which is the Cassie-Baxter model in eq 4 . When all grooves are preoccupied by gas and can be penetrated by liquid, i.e., $t_{1}=1-\phi_{\mathrm{S}}$ and $t_{2}=0\left(q_{1}=1-\phi_{\mathrm{S}}\right.$ and $q_{2}=q_{3}=q_{4}=0$ in Figure 2), the model can be reduced as the Wenzel model $\cos \theta^{*}$ $=r \cos \theta_{\mathrm{o}}$, given in eq 3 . If all grooves are occupied by liquid and cannot be penetrated by gas, i.e., $t_{1}=0$ and $t_{2}=\phi_{\mathrm{S}}-1\left(q_{4}=1-\right.$ $\phi_{\mathrm{S}}$ and $q_{1}=q_{2}=q_{3}=0$ in Figure 2), the model turns into the impregnating model $\cos \theta^{*}=\phi_{\mathrm{S}} \cos \theta_{\mathrm{o}}+1-\phi_{\mathrm{S}}$, as given in eq 5 . Under given conditions, the model presented in this work can turn into the existed models mentioned above. The feasible region of $t_{1}$ and $t_{2}$ is demonstrated in Figure 3 and the three existing models are three special points located at axes.

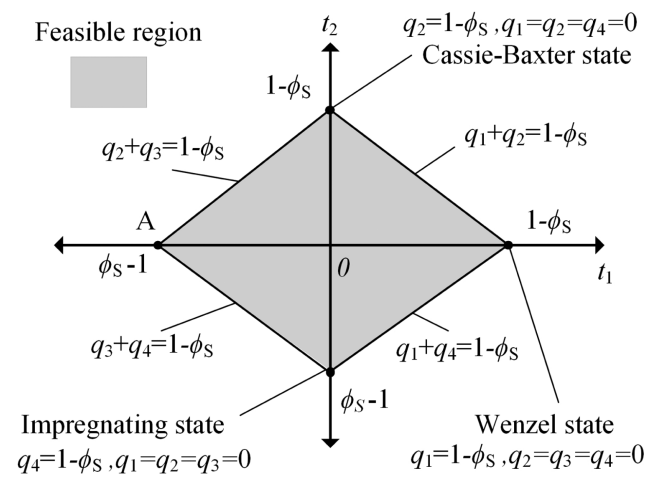

Figure 3. Feasible region for variable $t_{1}$ and $t_{2}$.

An unreported metastable contact angle under extreme conditions may be found in the feasible region, which is marked as point $A$ in Figure 3. The necessary and sufficient conditions for point $\mathrm{A}$ is $t_{1}=\phi_{\mathrm{S}}-1$ and $t_{2}=0\left(q_{1}-q_{3}=\phi_{\mathrm{S}}-1\right.$ and $q_{2}-q_{4}$ $=0)$. There is one sufficient and unnecessary, but realistic condition for point A. When $q_{3}=1-\phi_{\mathrm{S}}$ and $q_{1}=q_{2}=q_{4}=0$, indicating that all grooves are preoccupied by liquid and can be penetrated by gas, the metastable contact angle of point A may occur. Substituting $t_{1}=\phi_{\mathrm{S}}-1$ and $t_{2}=0$ into eq $16, \cos \theta^{*}=$ $\left(2 \phi_{\mathrm{S}}-r\right) \cos \theta_{\mathrm{o}}$ is obtained. These conditions are featured with the tendency of gas totally displacing liquid in the grooves, which seems to be the occurrence of the receding angle.

The boundaries of $t_{1}$ and $t_{2}$ feasible region are also special cases, which can be expressed by $\left|t_{1}\right|+\left|t_{2}\right|=1-\phi_{\mathrm{S}}$. Combining $q_{1}+q_{2}+q_{3}+q_{4}=1-\phi_{\mathrm{S}}$, the boundary can be reduced as $q_{1}+q_{2}$ $=1-\phi_{\mathrm{S}}$ when $t_{1}, t_{2}>0$; this boundary indicates that grooves are originally full of gas. When $t_{1}>0$ and $t_{2}<0$, the boundary conditions become $q_{1}+q_{4}=1-\phi_{\mathrm{S}}$, meaning the gas in the grooves can be totally displaced by liquid, but the liquid in the grooves cannot be displaced by gas. As for $t_{1}<0$ and $t_{2}<0$, the boundary conditions are reduced as $q_{3}+q_{4}=1-\phi_{\mathrm{S}}$, which means all grooves are preoccupied by liquid. When $t_{1}<0$ and $t_{2}>$ 0 , the boundary represents $q_{2}+q_{3}=1-\phi_{\mathrm{S}}$, meaning the gas in the grooves cannot be displaced by liquid, but the liquid in the grooves can be totally displaced by gas.

In all, the surface topography, the distribution of liquid/gas in surface defects (grooves), and the difficulty (probability) of liquid/gas mutual displacement influence the contact angle hysteresis. The introduction of variables $t_{1}$ and $t_{2}$ provides a way to explain the continuous change of the contact angle among multiple metastable states during hysteresis. The following section will introduce the estimation of variables $t_{1}$ and $t_{2}$ and obtain the function of advancing and receding angles.

2.3. Advancing and Receding Angles. 2.3.1. General Forms. The advancing angle is the maximum angle occurring during hysteresis. The corresponding parameters $t_{1}$ and $t_{2}$ are replaced by $t_{1 \mathrm{a}}$ and $t_{2 \mathrm{a}}$ and eq 16 can be rewritten as

$$
\cos \theta_{\mathrm{adv}}=\left(\phi_{\mathrm{S}}+t_{1 \mathrm{a}} \frac{r-\phi_{\mathrm{S}}}{1-\phi_{\mathrm{S}}}\right) \cos \theta_{\mathrm{o}}-t_{2 \mathrm{a}}
$$

The receding contact angle is the minimum angle of the metastable contact angle. ${ }^{27}$ As for the receding angle, we could use the same analysis for a quasi-static receding process, 
assuming that the three-phase point moves backward (gas displaces liquid) for a distance of $\mathrm{d} x$, as depicted in Figure 4.

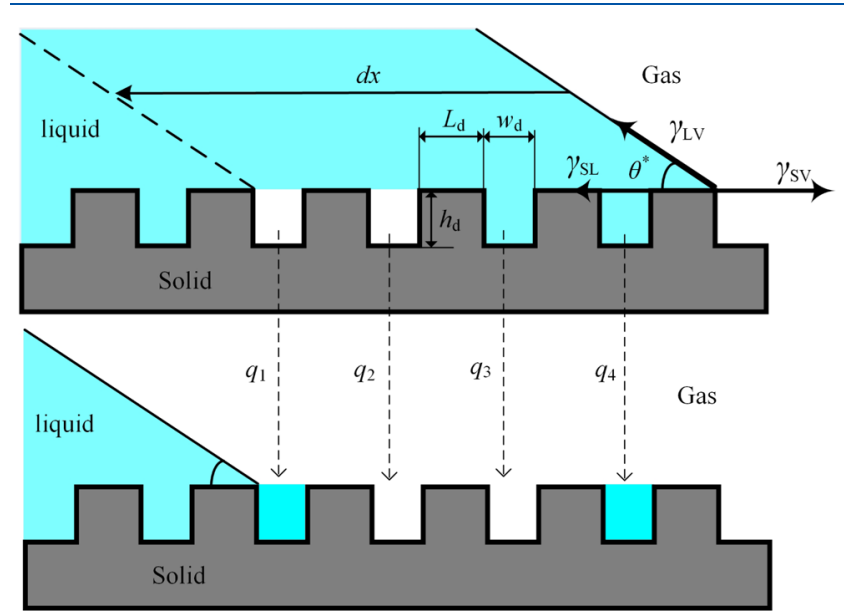

Figure 4. Surface topography, the fraction of gas and liquid prefilling the grooves and the fraction of gas and liquid displaced by the other phase, together influence the quasi-static contact angle during the contact point moving backward for the distance of $\mathrm{d} x$.

The change in surface energy involves:

1. Solid-liquid interfaces turn into solid-gas interfaces on the top of defects with the fraction of $\phi_{S}$. The change in surface energy is $-\phi_{\mathrm{S}}\left(\gamma_{\mathrm{SL}}-\gamma_{\mathrm{SV}}\right) \mathrm{d} x$.

2. During the movement, grooves originally occupied by gas will be penetrated by liquid, with the quantity of $q_{1}$. Solid-gas interfaces will be displaced by solid-liquid interfaces, and the change of surface energy is $q_{1}\left(1+2 h_{\mathrm{d}} /\right.$ $\left.w_{\mathrm{d}}\right)\left(\gamma_{\mathrm{SL}}-\gamma_{\mathrm{SV}}\right) \mathrm{d} x$; the fraction $q_{2}$ of gas-occupied grooves will remain unchanged and the new liquid-gas interfaces will form upon the grooves, the change in surface energy being $-q_{2} \gamma_{\mathrm{LV}} \mathrm{d} x$ (different from the advancing process).

3. Grooves originally occupied by liquid will be penetrated by gas, with the quantity of $q_{3}$. Solid-liquid interfaces will be displaced by solid-gas interfaces, and the change of surface energy is given by $-q_{3}\left(1+2 h_{\mathrm{d}} / w_{\mathrm{d}}\right)\left(\gamma_{\mathrm{SL}}-\gamma_{\mathrm{SV}}\right) \mathrm{d} x$; while the other part $q_{4}$ of liquid-occupied grooves will remain unchanged, but extra liquid-gas interfaces will form, the change of surface energy being $q_{4} \gamma_{\mathrm{LV}} \mathrm{d} x$ (different from the advancing process).

4. The work done by the liquid-gas interfacial tension $\gamma_{\mathrm{LV}}$ along the distance $\mathrm{d} x$, increase the surface energy by $-\gamma_{\mathrm{LV}}$ $\cos \theta^{*} \mathrm{~d} x$ (different from the advancing process).

The total change $d E$ in surface energy is as follows

$$
\begin{aligned}
\mathrm{d} E= & {\left[-\phi_{\mathrm{S}}+\left(q_{1}-q_{3}\right) \frac{2 h_{\mathrm{d}}+w_{\mathrm{d}}}{w_{\mathrm{d}}}\right]\left(\gamma_{\mathrm{SL}}-\gamma_{\mathrm{SV}}\right) \mathrm{d} x } \\
& -\left(q_{2}-q_{4}\right) \gamma_{\mathrm{LV}} \mathrm{d} x-\gamma_{\mathrm{LV}} \cos \theta^{*} \mathrm{~d} x
\end{aligned}
$$

The expression of $\theta_{\text {rec }}$ can be obtained from $\mathrm{d} E=0$. Displacing $h_{\mathrm{d}}$ and $w_{\mathrm{d}}$ with $r$ and $\phi_{\mathrm{S}}$ and letting $t_{1}=q_{1}-q_{3}$ and $t_{2}=q_{2}-q_{4}$, to reduce the expression. The receding angle is the minimum angle during hysteresis, and the corresponding parameters $t_{1}$ and $t_{2}$ are denoted as $t_{1 \mathrm{r}}$ and $t_{2 \mathrm{r}}$.

$$
\cos \theta_{\mathrm{rec}}=\left(\phi_{\mathrm{S}}-t_{1 \mathrm{r}} \frac{r-\phi_{\mathrm{S}}}{1-\phi_{\mathrm{S}}}\right) \cos \theta_{\mathrm{o}}-t_{2 \mathrm{r}}
$$

Comparing eqs 17 with 19 , it can be found that the sign before $t_{1 \mathrm{a}}$ and $t_{1 \mathrm{r}}$ are opposite. $t_{1 \mathrm{a}}$ and $t_{1 \mathrm{r}}$ stand for the same physical meaning, but they exert varied influence on $\theta_{\text {adv }}$ and $\theta_{\text {rec }}$. The occurrence of advancing and receding angles can be attributed to the same physical mechanism, which contributes differently to the surface energy, as discussed above. This is the source of difference between the advancing and receding angles. Now $\theta_{\mathrm{adv}}$ and $\theta_{\text {rec }}$ 's function of $\theta_{\mathrm{o}}$ is obtained as shown below.

$$
\left\{\begin{array}{l}
\cos \theta_{\mathrm{adv}}=\left(\phi_{\mathrm{S}}+t_{1 \mathrm{a}} \frac{r-\phi_{\mathrm{S}}}{1-\phi_{\mathrm{S}}}\right) \cos \theta_{\mathrm{o}}-t_{2 \mathrm{a}} \\
\cos \theta_{\mathrm{rec}}=\left(\phi_{\mathrm{S}}-t_{1 \mathrm{r}} \frac{r-\phi_{\mathrm{S}}}{1-\phi_{\mathrm{S}}}\right) \cos \theta_{\mathrm{o}}-t_{2 \mathrm{r}}
\end{array}\right.
$$

In eq 20, the physical meanings of four parameters $t_{1 a}, t_{2 a}, t_{1 \mathrm{r}}$, and $t_{2 \mathrm{r}}$ have been discussed above. The estimations of the four parameters will be discussed in the following section.

2.3.2. Parameter Estimations. The surface topography can be analyzed by a scanning electron microscope ${ }^{10}$ and an atomic force microscope. ${ }^{11,12}$ Some researchers use solid decorated with microstructures (pillars or holes) to control the surface roughness. ${ }^{17,28,29}$ Thus, $\phi_{\mathrm{S}}$ and $r$ can be directly measured or controlled in experiments. However, there is no direct measurement of $t_{1 a}, t_{2 a}, t_{1 \mathrm{r}}$, and $t_{2 \mathrm{r}}$. It cannot be assumed that the different three-phase system shares the same parameters. Furthermore, relating $t_{1}\left(t_{1 \mathrm{a}} / t_{1 \mathrm{r}}\right)$ and $t_{2}\left(t_{2 \mathrm{a}} / t_{2 \mathrm{r}}\right)$ with all other parameters like $\phi_{\mathrm{S}}, r$, and $\theta_{\mathrm{o}}$, would add more unknown parameters to the model, and make it complex to verify the model. Here, we propose simple linear functions to estimate $t_{1}$ and $t_{2}$. Since $\left|t_{1}\right|+\left|t_{2}\right| \leq 1-\phi_{S}, t_{1}$ and $t_{2}$ might increase with the rise of $1-\phi_{\mathrm{S}}, t_{1}, t_{2} \propto 1-\phi_{\mathrm{S}}$. $t_{1}$ is related to the fraction of grooves penetrated by liquid. The smaller the contact angle $\theta^{*}$, the stronger the tendency (wettability) for the liquid to cover the solid surface. Thus, it is assumed that $t_{1} \propto \cos \theta^{*}+1$. The constant one in the expression is used to obtain a non-negative result. In contrast, $t_{2}$ is related to the fraction of residual gas in the grooves. The contact angle on the gas side $\pi-\theta^{*}$ reflects the tendency of gas covering the solid surface. Thus, it is assumed that $t_{2} \propto \cos \left(\pi-\theta^{*}\right)+1=1-\cos \theta^{*}$. If $\theta^{*}$ is replaced by the advancing and receding angles, linear functions for $t_{1}\left(t_{1 \mathrm{a}} / t_{1 \mathrm{r}}\right)$ and $t_{2}\left(t_{2 \mathrm{a}} / t_{2 \mathrm{r}}\right)$ can be obtained

$$
\begin{gathered}
\left\{\begin{array}{l}
t_{1 \mathrm{a}}=K_{\mathrm{a}}^{\prime}\left(1-\phi_{\mathrm{S}}\right)\left(\cos \theta_{\mathrm{adv}}+1\right) \\
t_{2 \mathrm{a}}=K_{\mathrm{a}}^{\prime \prime}\left(1-\phi_{\mathrm{S}}\right)\left(1-\cos \theta_{\mathrm{adv}}\right)
\end{array}\right. \\
\left\{\begin{array}{l}
t_{1 \mathrm{r}}=K_{\mathrm{r}}^{\prime}\left(1-\phi_{\mathrm{S}}\right)\left(\cos \theta_{\mathrm{rec}}+1\right) \\
t_{2 \mathrm{r}}=K_{r}^{\prime \prime}\left(1-\phi_{\mathrm{S}}\right)\left(1-\cos \theta_{\mathrm{rec}}\right)
\end{array}\right.
\end{gathered}
$$

where $K_{\mathrm{a}}^{\prime}, K_{\mathrm{a}}^{\prime \prime}, K_{\mathrm{r}}^{\prime}$, and $K_{\mathrm{r}}^{\prime \prime}$ are the factors of the linear functions. Substituting eqs 21 into eq 20, the final expression for $\theta_{\text {adv }}$ and $\theta_{\text {rec }}$ can be obtained.

$$
\left\{\begin{array}{l}
\cos \theta_{\mathrm{adv}}=\frac{\left[\phi_{\mathrm{S}}+K_{\mathrm{a}}^{\prime}\left(r-\phi_{\mathrm{S}}\right)\right] \cos \theta_{\mathrm{o}}-K_{\mathrm{a}}^{\prime \prime}\left(1-\phi_{\mathrm{S}}\right)}{1-K_{\mathrm{a}}^{\prime}\left(r-\phi_{\mathrm{S}}\right) \cos \theta_{\mathrm{o}}-K_{\mathrm{a}}^{\prime \prime}\left(1-\phi_{\mathrm{S}}\right)} \\
\cos \theta_{\mathrm{rec}}=\frac{\left[\phi_{\mathrm{S}}-K_{\mathrm{r}}^{\prime}\left(r-\phi_{\mathrm{S}}\right)\right] \cos \theta_{\mathrm{o}}-K_{\mathrm{r}}^{\prime \prime}\left(1-\phi_{\mathrm{S}}\right)}{1+K_{\mathrm{r}}^{\prime}\left(r-\phi_{\mathrm{S}}\right) \cos \theta_{\mathrm{o}}-K_{\mathrm{r}}^{\prime \prime}\left(1-\phi_{\mathrm{S}}\right)}
\end{array}\right.
$$



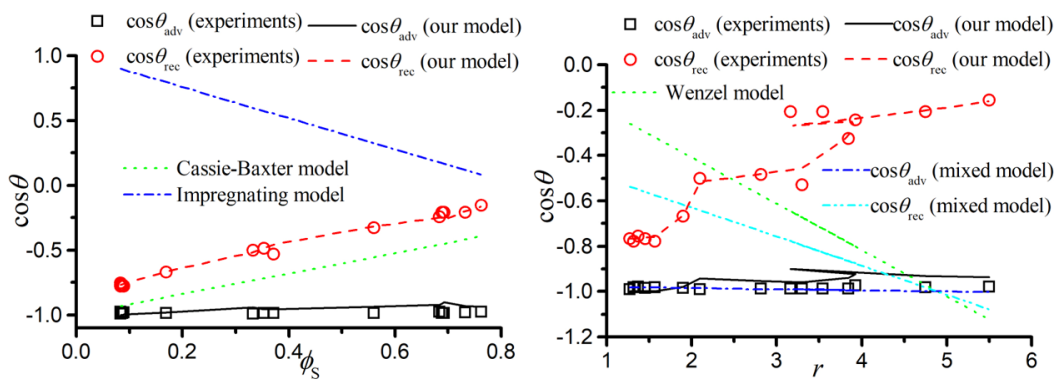

(a) Water results
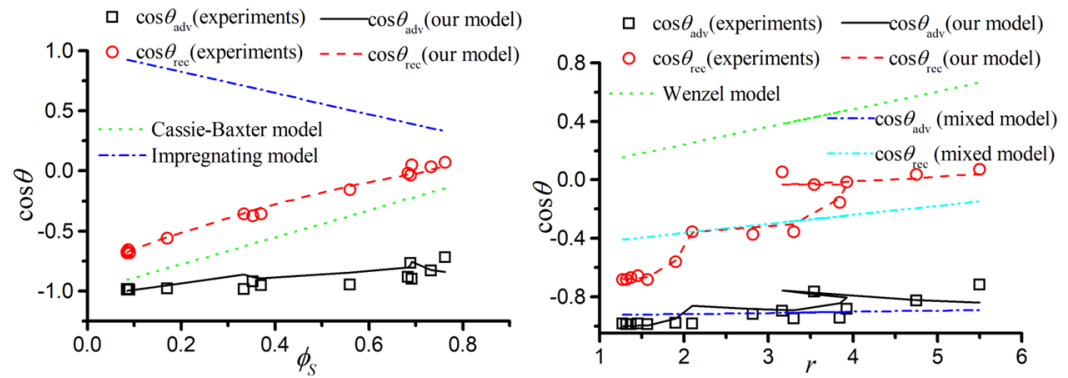

(b) Ethylene glycol results

Figure 5. Comparison between models and experiments of Koch et al.

Equation 21 provides possible expressions for $t_{1 a}, t_{2 a}, t_{1 \mathrm{r}}$, and $t_{2 \mathrm{r}}$. No matter how these four parameters are correlated with other physical parameters, they have to satisfy

$$
\left|t_{1}\right|+\left|t_{2}\right| \leq 1-\phi_{S}
$$

To verify the model, eq 22 will be compared with experiments and the fitting will be carried out under the constraint in eq 23 .

\section{COMPARISON WITH EXPERIMENTS AND DISCUSSIONS}

3.1. Experiments with Known $\phi_{\mathrm{S}}$ and $r$. Some experiments concerning contact angle hysteresis measure the advancing and receding angles of the same liquid, on different solid plates. The parameters $\phi_{\mathrm{S}}$ and $r$ are directly measured or controlled in these experiments. ${ }^{23,26,29}$ The model presented in this work needs to fit four parameters $K_{\mathrm{a}}^{\prime}, K_{\mathrm{a}}^{\prime \prime}, K_{\mathrm{r}}^{\prime}$, and $K_{\mathrm{r}}^{\prime \prime}$ in comparison with experiments. $\phi_{\mathrm{S}}$ and $r$ are directly given or calculated by the method given by each experiment. Experiments also provide measured values of advancing angles $\theta_{\mathrm{adv}}^{\exp }$ receding angles $\theta_{\text {rec }}^{\exp }$, and Young contact angles $\theta_{\mathrm{o}}$. The nonlinear optimization methods in MATLAB are used to solve for $K_{\mathrm{a}}^{\prime}, K_{\mathrm{a}}^{\prime \prime}$, $K_{\mathrm{r}}^{\prime}$, and $K_{\mathrm{r}}^{\prime \prime}$ in eq 24 , wherein the fitting advancing and receding angles $\theta_{\mathrm{adv}}^{\mathrm{fit}}$ and $\theta_{\mathrm{rec}}^{\mathrm{fit}}$ are calculated from $K_{\mathrm{a}}^{\prime}, K_{\mathrm{a}}^{\prime \prime}, K_{\mathrm{r}}^{\prime}$, and $K_{\mathrm{r}}^{\prime \prime}$.

$$
\begin{aligned}
& \min _{K^{\prime}, K^{\prime \prime}}(1-\alpha) \frac{\sum_{1}^{n}\left(\cos \theta^{\exp }-\cos \theta^{\mathrm{fit}}\right)^{2}}{n} \\
& \quad+\alpha\left|\left(\left|t_{1}\right|+\left|t_{2}\right|\right)_{\max }-1+\phi_{S}\right| \\
& \text { s. t. }\left\{\begin{array}{l}
\cos \theta^{\mathrm{fit}}-f\left(K^{\prime}, K^{\prime \prime}\right)=0 \\
t_{1}-K^{\prime}\left(1-\phi_{S}\right)\left[f\left(K^{\prime}, K^{\prime \prime}\right)+1\right]=0 \\
t_{2}-K^{\prime \prime}\left(1-\phi_{S}\right)\left[-f\left(K^{\prime}, K^{\prime \prime}\right)+1\right]=0 \\
K^{\prime} \leq 100 \\
\left|K^{\prime \prime}\right| \leq 100
\end{array}\right.
\end{aligned}
$$

In eq $24, K^{\prime}$ represents $K_{\mathrm{a}}^{\prime}$ or $K_{\mathrm{r}}^{\prime}$; $K^{\prime \prime}$ represents $K_{\mathrm{a}}^{\prime \prime}$ or $K_{\mathrm{r}}^{\prime \prime}$; $\alpha$ is a non-negative factor less than unity, adjusting fitting objectives; $n$ is the number of experimental data; $\theta^{\exp }$ represents $\theta_{\text {adv }}^{\exp }$ or $\theta_{\text {rec }}^{\exp } t_{1}$ denotes $t_{1 \mathrm{a}}$ or $t_{1 \mathrm{r}} ; t_{2}$ denotes $t_{2 \mathrm{a}}$ or $t_{2 \mathrm{r}}$; and $f\left(K^{\prime}, K^{\prime \prime}\right)$ is the expression for advancing angle or receding angle in eq 22 . The solutions of $\theta_{\mathrm{adv}}^{\mathrm{fit}}$ and $\theta_{\mathrm{rec}}^{\mathrm{fit}}$ have to be close to $\theta_{\mathrm{adv}}^{\exp }$ and $\theta_{\mathrm{rec}}^{\text {exp }}$, respectively, on one hand. On the other hand, $t_{1}$ and $t_{2}$ have to satisfy the constraint of eq 23 as much as possible for all measured data. The fitting objective is adjusted by factor $\alpha$ for accepted solutions, which is between $10^{-3}$ and $10^{-2}$.

3.1.1. Experiments of Koch et al. Koch et al. measured the advancing and receding angles of water and ethylene glycol on different solid surfaces. ${ }^{23}$ Solid surfaces are fabricated with micropillars with different diameters and inter-distances. The height of all pillars is $30 \mu \mathrm{m}$. The parameter $\phi_{\mathrm{S}}$ of a given solid surface is calculated by the pillars' diameter $d$ and inter-distance $s$ in a hexagonal unit cell ${ }^{23}$

$$
\phi_{\mathrm{S}}=\frac{\pi d^{2}}{\sqrt{12}(d+s)^{2}}
$$

In the same unit cell, the parameter $r$ can be calculated by

$$
r=1+\frac{2 \pi d h_{\mathrm{d}}}{3^{1 / 2}(d+s)^{2}}
$$

The Young contact angle $\theta_{\mathrm{o}}$ of a certain liquid is calculated by the measured advancing and receding angles $\theta_{\mathrm{adv}}^{\text {smooth }}$ and $\theta_{\text {rec }}^{\text {smooth }}$ on a smooth solid sample. ${ }^{23}$

$$
\cos \theta_{\mathrm{o}}=\frac{1}{2}\left(\cos \theta_{\mathrm{adv}}^{\text {smooth }}+\cos \theta_{\mathrm{rec}}^{\text {smooth }}\right)
$$

The comparison between models and water/ethylene glycol results are demonstrated in Figure 5. When comparing the relationship between $\cos \theta_{\text {adv }}\left(\cos \theta_{\text {rec }}\right)$ and $\phi_{\mathrm{S}}$, the CassieBaxter model, the impregnating model, and our model are involved in comparison. When comparing the relationship between $\cos \theta_{\text {adv }}\left(\cos \theta_{\text {ec }}\right)$ and $r$, the Wenzel model, the mixedstate model, and our model are involved. The Wenzel, Cassie- 
Table 1. Fitted Parameters for the Experiments of Koch et al.

\begin{tabular}{|c|c|c|c|c|c|c|c|c|}
\hline liquid & $K_{\mathrm{a}}^{\prime}$ & $K_{\mathrm{a}}^{\prime \prime}$ & $K_{\mathrm{r}}^{\prime}$ & $K_{\mathrm{r}}^{\prime \prime}$ & $\left(\frac{t_{1 \mathrm{a}}}{1-\phi_{\mathrm{S}}}\right)$ & $\left(\frac{t_{2 \mathrm{a}}}{1-\phi_{\mathrm{S}}}\right)$ & $\overline{\left(\frac{t_{1 \mathrm{r}}}{1-\phi_{\mathrm{S}}}\right)}$ & $\left(\frac{t_{2 \mathrm{r}}}{1-\phi_{\mathrm{S}}}\right.$ \\
\hline water & 8.9 & 0.5 & 0.2 & 0.5 & 0.36 & 1.05 & 0.08 & 0.70 \\
\hline ethylene glycol & -7.5 & 0.6 & -0.1 & 0.5 & -0.78 & 1.05 & -0.06 & 0.60 \\
\hline
\end{tabular}

(a) Micro-pillar results

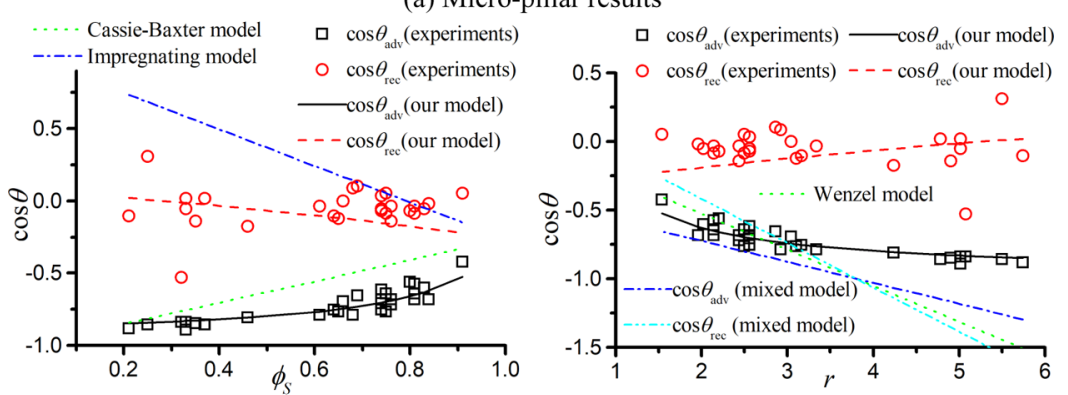

(b) Micro-hole results
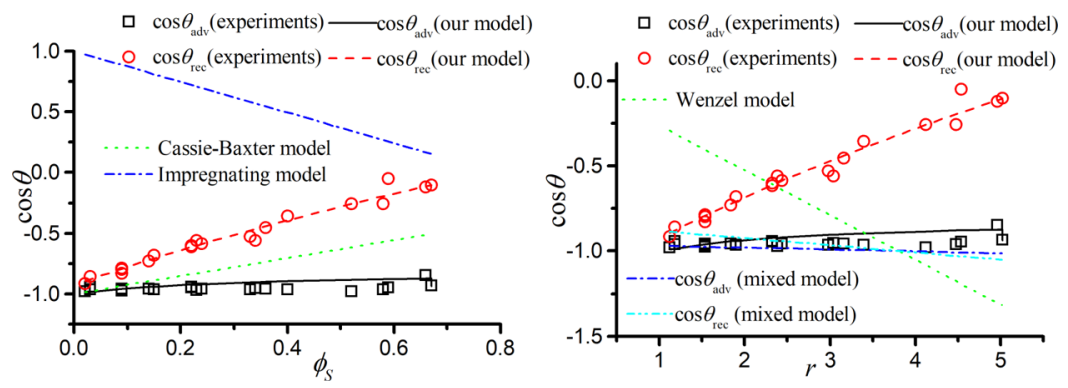

Figure 6. Comparison between models and experiments of Priest et al.

Baxter, and impregnating models can be directly calculated by given $\phi_{\mathrm{S}}$ and $r$. The mixed-state model needs fitting for an unknown parameter $\phi_{\mathrm{S}}^{\prime}$ in eq 6 . As shown in Figure 5, the experimental data are in good coincidence with the model presented in this work. However, the Wenzel, impregnating, and mixed-state models show obvious deviation from experiments. The Cassie-Baxter model predicts an acceptable trend but also shows deviation from experiments. The deviation indicates that the presumed states of these models might be incorrect under the experimental conditions.

The mixed-wetting model assumes a variable solid fraction $\phi_{S}^{\prime}$. The advancing and receding angles are fitted by the mixedwetting model separately, the best fitting result of which gives $\phi_{S}^{\prime}$ $=0.025$ for advancing and $\phi_{S}^{\prime}=0.624$ for receding. The mixedwetting model is also in good coincidence with the advancing angles but shows a wrong trend for the receding angles, as shown in Figure 5a. There is only one parameter to be fitted, which limits its ability to describe the nonlinear trend of the experimental $\cos \theta / r$ data, as depicted in Figure $5 \mathrm{~b}$. The nonlinear trend is induced by the coordinated influence of $\phi_{\mathrm{S}}$ and $r$ because these two variables are simultaneously changed in experiments. The model proposed in this work has accounted for $\phi_{\mathrm{S}}$ and $r$ and fits better the nonlinear trend, as shown in Figure 5.

Table 1 lists the average ratio of $t_{1 \mathrm{a}}\left(t_{1 \mathrm{r}}, t_{2 \mathrm{a}}\right.$ and $\left.t_{2 \mathrm{r}}\right)$ to $\phi_{\mathrm{S}}$, which reflects if the fitting results adequately follow the constraint of $\left|t_{1}\right|$ $+\left|t_{2}\right| \leq 1-\phi_{\mathrm{S}}$. As for advancing angles, $t_{1 \mathrm{a}}$ and $t_{2 \mathrm{a}}$ are not negligible, meaning the filler in the grooves is likely to change, either from liquid to gas or from gas to liquid. However, the probability of gas remaining in grooves is higher than that of liquid. The $t_{1 \mathrm{r}}$ of both liquids are small enough to be neglected.
This means the fraction of gas displaced by liquid almost equals the fraction of liquid displaced by gas. Such conditions would get close to the Cassie-Baxter state if $t_{2 \mathrm{r}}$ approximates $1-\phi_{\mathrm{S}}$. The fitting results of receding angles are well within the fitting constraint, but the results of advancing angles are out of limits. I $t_{1 \mathrm{a}}|+| t_{2 \mathrm{a}} \mid$ exceeds the limit of $1-\phi_{\mathrm{S}}$ by $41 \%$ for water and $83 \%$ for ethylene glycol. Complete fitting results are given in the Supporting Information.

3.1.2. Experiments of Priest et al. Priest et al. fabricated square micropillars or microholes on the solid surface to control the parameters of $\phi_{\mathrm{S}}$ and $r{ }^{29}$ The widths of pillars and holes are $w=20 \mu \mathrm{m}$, heights $h=30 \mu \mathrm{m}$. $\phi_{\mathrm{S}}$ and $r$ are controlled by adjusting the inter-distance $d$ between microstructures. For square micropillars, $\phi_{\mathrm{S}}=w^{2} / d^{2}$; for square microholes, $\phi_{\mathrm{S}}=1-$ $w^{2} / d^{2}{ }^{29}$ For both structures, $r$ can be calculated by

$$
r=1+\frac{4 w h}{d^{2}}
$$

Water is the only test liquid and its advancing and receding angles on the smooth surface are $\theta_{\mathrm{adv}}^{\text {smooth }}=72^{\circ}$ and $\theta_{\text {rec }}^{\text {smoth }}=59^{\circ}$. The Young contact angle can be computed by eq 27 .

The comparison between theoretical models and experiments on solid with micropillars is demonstrated in Figure 6a. Our model is in good coincidence with both advancing and receding angles. The impregnating model and the Wenzel model predict the wrong trend of $\cos \theta$. The Cassie-Baxter model predicts the right trend but still shows obvious deviation from both advancing and receding angles. The results of microholes are demonstrated in Figure 6b. The Wenzel, Cassie-Baxter, and impregnating models have intersections with the experiment. However, the model presented in this work adequately fit the 
Table 2. Fitted Parameters for the Experiments of Priest et al.

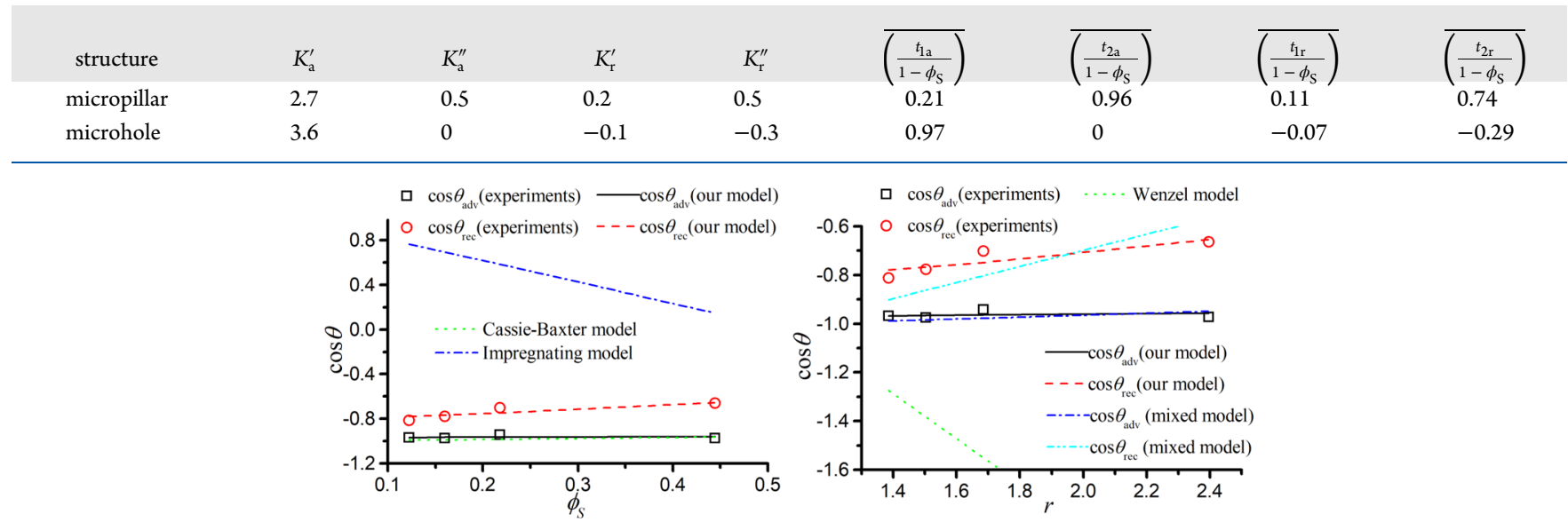

Figure 7. Comparison between models and experiments of Yeong et al.

Table 3. Fitted Parameters for the Experiments of Yeong et al.

\begin{tabular}{|c|c|c|c|c|c|c|c|c|}
\hline parameter & $K_{\mathrm{a}}^{\prime}$ & $K_{\mathrm{a}}^{\prime \prime}$ & $K_{\mathrm{r}}^{\prime}$ & $K_{\mathrm{r}}^{\prime \prime}$ & $\left(\frac{t_{1 \mathrm{a}}}{1-\phi_{\mathrm{S}}}\right)$ & $\left(\frac{t_{2 \mathrm{a}}}{1-\phi_{\mathrm{S}}}\right)$ & $\left(\frac{t_{1 \mathrm{r}}}{1-\phi_{\mathrm{S}}}\right)$ & $\frac{t_{2 \mathrm{r}}}{1-\phi_{\mathrm{S}}}$ \\
\hline & 0.2 & 0.5 & 0.3 & 0.5 & 0.01 & 0.97 & 0.08 & 0.83 \\
\hline
\end{tabular}

nonlinear trend of $\cos \theta_{\text {adv }}$, the fitting results of which are close to the Cassie-Baxter state for advancing angles $\left(t_{1 \mathrm{a}} \approx 1-\phi_{\mathrm{S}}, t_{2 \mathrm{a}}=\right.$ $0)$. The best-fitting results of the mixed-wetting model are demonstrated in Figure 6a,b. For receding angles, the mixed model predicts the wrong trend, due to the limit of only one fitted parameter.

Table 2 lists the average values of normalized $t_{1 a}, t_{1 \mathrm{r}}, t_{2 \mathrm{a}}$ and $t_{2 \mathrm{r}}$. The fitting results for the advancing angle on the micropillars surface slightly go beyond the constraint, $\left|t_{1 \mathrm{a}}\right|+\left|t_{2 \mathrm{a}}\right|$, exceeding the limit of $1-\phi_{\mathrm{S}}$ by around $10 \%$. Other fitting results are well within the limit. Complete fitting values of model parameters are given in the Supporting Information.

3.1.3. Experiments of Yeong et al. Yeong et al. measured the advancing and receding angles of water on solid surfaces fabricated with square micropillars. ${ }^{29}$ The patterns of micropillars are the same as the experiments conducted by Priest et al. Thus, $\phi_{\mathrm{S}}$ and $r$ are computed by the same method as mentioned above. However, the Young contact angle is not given in the paper. Here, the average value of apparent contact angles measured on different surfaces is taken as the Young contact angle $\theta_{\mathrm{o}}$. The comparison is shown in Figure 7 , where the Cassie-Baxter model and our model give the best fitting, while other models predict wrong trends and exhibit obvious deviation. It can also be found that the mixed-wetting model is in good coincidence with the advancing angles and shows deviation from the receding angles.

From Table 3, it can be found that the fitting results of advancing and receding are quite close to the state of CassieBaxter $\left(t_{1 \mathrm{a}} \approx 0, t_{2 \mathrm{a}} \approx 1-\phi_{\mathrm{S}}\right)$. All results well satisfy the constraint of $\left|t_{1}\right|+\left|t_{2}\right| \leq 1-\phi_{S}$. The fitting results of model parameters are given in the Supporting Information.

3.1.4. Experiments with Unknown $\phi_{s}$ and $r$. Another series of experiments measure the advancing and receding angles of different liquids on the same solid plate. ${ }^{9,30}$ These experiments do not necessarily provide information about $\phi_{\mathrm{S}}$ and $r$. Thus, there are six parameters needing fitting in comparison with these experiments. Usually, the Young contact angle is not provided. For the convenience of discussion, the relationship between $\cos \theta_{\text {adv }}$ and $\cos \theta_{\text {rec }}$ is used for comparison. Eliminating $\theta_{\mathrm{o}}$ in eq 22 yields

$$
\cos \theta_{\mathrm{rec}}=\frac{\left[\phi_{\mathrm{S}}-K_{\mathrm{r}}^{\prime}\left(r-\phi_{\mathrm{S}}\right)\right] F\left(\theta_{\mathrm{adv}}\right)-K_{\mathrm{r}}^{\prime \prime}\left(1-\phi_{\mathrm{S}}\right)}{1+K_{\mathrm{r}}^{\prime}\left(r-\phi_{\mathrm{S}}\right) F\left(\theta_{\mathrm{adv}}\right)-K_{\mathrm{r}}^{\prime \prime}\left(1-\phi_{\mathrm{S}}\right)}
$$

wherein $F\left(\theta_{\text {adv }}\right)$ is given by eq 30 .

$$
F\left(\theta_{\mathrm{adv}}\right)=\frac{\cos \theta_{\mathrm{adv}}+K_{\mathrm{a}}^{\prime \prime}\left(1-\phi_{\mathrm{S}}\right)\left(1-\cos \theta_{\mathrm{adv}}\right)}{\phi_{\mathrm{S}}+K_{\mathrm{a}}^{\prime}\left(r-\phi_{\mathrm{S}}\right)\left(1+\cos \theta_{\mathrm{adv}}\right)}
$$

The nonlinear optimization methods in MATLAB are used to solve for $\phi_{\mathrm{S}}, r, K_{\mathrm{a}}^{\prime}, K_{\mathrm{a}}^{\prime \prime}, K_{\mathrm{r}}^{\prime}$, and $K_{\mathrm{r}}^{\prime \prime}$ in eq 31 .

$$
\begin{aligned}
& \min _{\phi_{\mathrm{S}}, r, K_{\mathrm{a}}^{\prime}, K_{\mathrm{a}}^{\prime \prime}, K_{\mathrm{r}}^{\prime}, K_{\mathrm{r}}^{\prime \prime}}(1-\alpha) \frac{\sum_{1}^{n}\left(\cos \theta_{\mathrm{rec}}^{\exp }-\cos \theta_{\mathrm{rec}}^{\mathrm{fit}}\right)^{2}}{n} \\
& +\frac{\alpha}{2}\left|\left(\left|t_{1 \mathrm{a}}\right|+\left|t_{2 \mathrm{a}}\right|\right)_{\max }-1+\phi_{\mathrm{S}}\right| \\
& +\frac{\alpha}{2}\left|\left(\left|t_{1 \mathrm{r}}\right|+\left|t_{2 \mathrm{r}}\right|\right)_{\max }-1+\phi_{\mathrm{S}}\right| \\
& \text { s. t. }\left\{\begin{array}{l}
\cos \theta_{\mathrm{rec}}^{\mathrm{fit}}-f_{1}\left(\phi_{\mathrm{S}}, r, K_{\mathrm{a}}^{\prime}, K_{\mathrm{a}}^{\prime \prime}, K_{\mathrm{r}}^{\prime}, K_{\mathrm{r}}^{\prime \prime}, \theta_{\mathrm{adv}}^{\exp }\right)=0 \\
t_{1 \mathrm{a}}-K_{\mathrm{a}}^{\prime}\left(1-\phi_{\mathrm{S}}\right)\left[\cos \theta_{\mathrm{adv}}^{\exp }+1\right]=0 \\
t_{2 \mathrm{a}}-K_{\mathrm{a}}^{\prime \prime}\left(1-\phi_{\mathrm{S}}\right)\left[-\cos \theta_{\mathrm{adv}}^{\exp }+1\right]=0 \\
t_{1 \mathrm{r}}-K_{\mathrm{r}}^{\prime}\left(1-\phi_{\mathrm{S}}\right)\left[\cos \theta_{\mathrm{rec}}^{\mathrm{fit}}+1\right]=0 \\
t_{2 \mathrm{r}}-K_{\mathrm{r}}^{\prime \prime}\left(1-\phi_{\mathrm{S}}\right)\left[-\cos \theta_{\mathrm{rec}}^{\mathrm{fit}}+1\right]=0 \\
\left|K_{\mathrm{a}}^{\prime}\right| \leq 100,\left|K_{\mathrm{a}}^{\prime \prime}\right| \leq 100 \\
\left|K_{\mathrm{r}}^{\prime}\right| \leq 100,\left|K_{\mathrm{r}}^{\prime \prime}\right| \leq 100
\end{array}\right.
\end{aligned}
$$

In eq $31, \theta_{\text {rec }}^{\exp }$ stands for the measured data of receding angles; $\theta_{\mathrm{rec}}^{\mathrm{fit}}$ is the fitting values of receding angles; $\theta_{\mathrm{adv}}^{\exp }$ is the measured data of advancing angles; $\alpha$ is a non-negative factor less than unity, adjusting fitting objectives; $n$ is the number of 

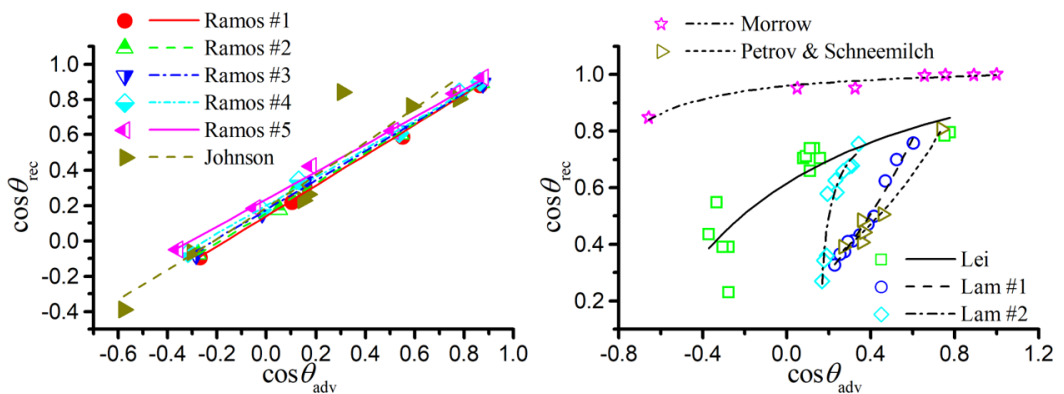

Figure 8. Comparison with experiments with unknown $\phi_{\mathrm{S}}$ and $r$. Points of different colors and shapes represent measured data. Lines represent the fitting results of the model presented in this work.

\section{Table 4. Height of the Nanoparticle ${ }^{12}$ and the Height of a Defect Computed by Fitting Results}

$\begin{array}{ccccccc}\text { identifier } & \phi_{\mathrm{S}} & r & \text { area density } / \times 10^{9} \cdot \mathrm{cm}^{-2} & L_{\mathrm{d}}+w_{\mathrm{d}} / \mathrm{nm} & \text { height of a particle } / \mathrm{nm} \\ \# 2 & 0.90 & 1.050 & 1 & 316 & 183 & 3 \\ \# 3 & 0.89 & 1.056 & 3 & 6 & 129 & 3 \\ \# 4 & 0.87 & 1.066 & 10 & 100 & 3.9 \\ \# 5 & 0.84 & 1.084 & 4.3 & 3\end{array}$
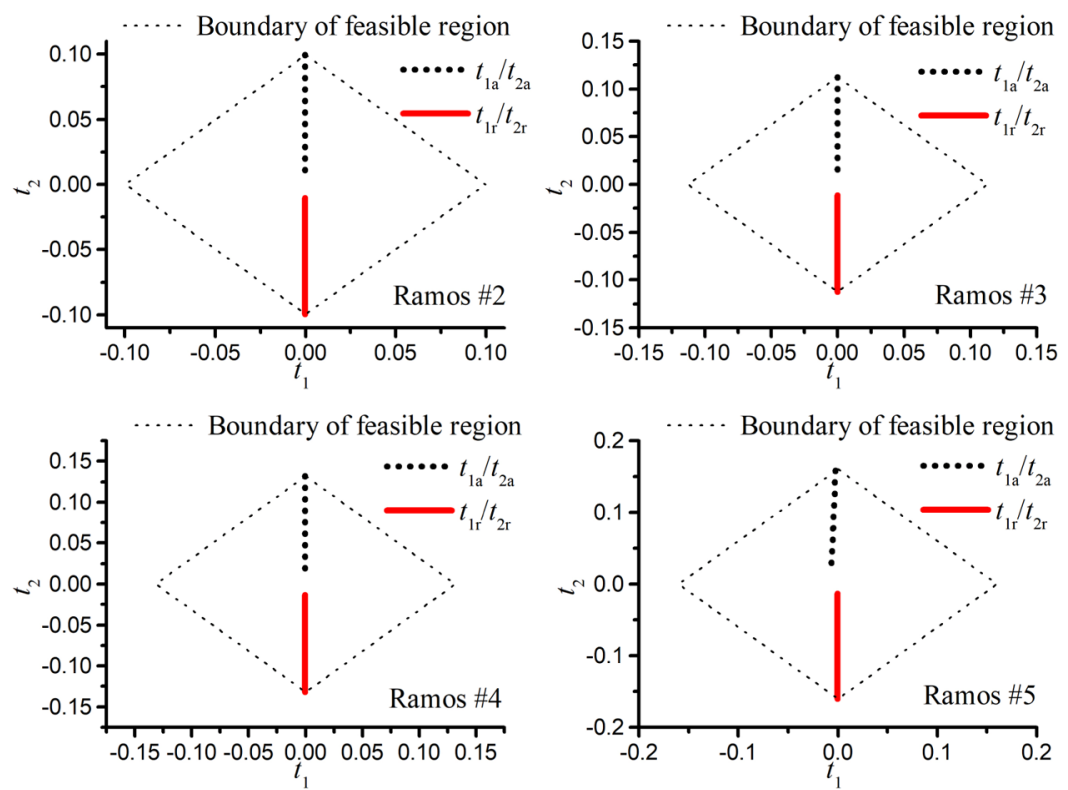

Figure 9. Distribution of $t_{1 \mathrm{a}} / t_{1 \mathrm{r}}$ and $t_{2 \mathrm{a}} / t_{2 \mathrm{r}}$ for the experiments of Ramos and Tanguy. ${ }^{12}$

experimental data; and $f_{1}$ represents the expression of the receding angle in eq 29.

Since there are 6 undetermined parameters, at least 6 measured data are needed for nonlinear fitting. Eleven groups of experiments are selected for the comparison with our model, as displayed in Figure 8. ${ }^{4,9,12,30-33}$ The model presented in this work fits well both the nonlinear and linear relationship of $\cos \theta_{\text {rec }} / \cos \theta_{\text {adv }}$.

The solid surfaces used by Ramos et al. are decorated with nanoscale particles, and the pristine surfaces are of $0.1 \mathrm{~nm}$ root mean square roughness. ${ }^{12}$ Nanoparticles are randomly distributed on the surface and the area density and geometry sizes are given in the paper. The number of particles $n$ within area $A$ is already known and the average wavelength $\left(L_{\mathrm{d}}+w_{\mathrm{d}}\right)$ of surface roughness can be calculated by

$$
\left(L_{\mathrm{d}}+w_{\mathrm{d}}\right)=\sqrt{\frac{A}{n-1}}
$$

According to eq 13 , the height of a defect $h_{\mathrm{d}}$ can be computed by $L_{\mathrm{d}}+w_{\mathrm{d}}$

$$
h_{\mathrm{d}}=\left(L_{\mathrm{d}}+w_{\mathrm{d}}\right)(r-1) / 2
$$

To verify the fitting results, the $h_{\mathrm{d}}$ computed by the fitting results are compared with the real height of nanoparticles. The comparison is listed in Table 4. The experimental solid surfaces are decorated with conical-shaped hillocks with a height of $3 \mathrm{~nm}$. The heights of a defect computed by fitting results range from 4.2 to $7.9 \mathrm{~nm}$. Our model presumes a rectangular shape of a defect, which deviates from the experimental conditions. Since the real height of a nanoparticle is of the same magnitude with the computed results, the fitting results of $r$ are acceptable.

The fitting $t_{1 a}, t_{1 \mathrm{r}}, t_{2 a}$, and $t_{2 \mathrm{r}}$ of Ramos' experiments are shown in Figure 9. Fitting results of other experiments can be found in the Supporting Information. The distribution of $t_{1 a}, t_{1 \mathrm{r}}, t_{2 a}$ and $t_{2 \mathrm{r}}$ are well within the feasible region of the model. It should be noted that some experiments present an obvious linear 
relationship between $\cos \theta_{\text {rec }}$ and $\cos \theta_{\text {adv }}$ and their fitting parameters $t_{1 \mathrm{a}} \approx t_{1 \mathrm{r}} \approx 0\left(K_{\mathrm{a}}^{\prime} \approx K_{\mathrm{r}}^{\prime} \approx 0\right)$. When $t_{1 \mathrm{a}}=t_{1 \mathrm{r}}=0$, the fraction of gas displaced by liquid in grooves equals the fraction of liquid displaced by gas in grooves. Substituting $t_{1 \mathrm{a}}=t_{1 \mathrm{r}}=0$ (i.e., $K_{\mathrm{a}}^{\prime}=K_{\mathrm{r}}^{\prime}=0$ ) into eq 29 yields

$$
\cos \theta_{\text {rec }}=\frac{K_{\mathrm{a}}^{\prime \prime}\left(1-\phi_{\mathrm{S}}\right)\left(1-\cos \theta_{\mathrm{adv}}\right)-K_{\mathrm{r}}^{\prime \prime}\left(1-\phi_{\mathrm{S}}\right)}{1-K_{\mathrm{r}}^{\prime \prime}\left(1-\phi_{\mathrm{S}}\right)}
$$

wherein $\cos \theta_{\text {rec }}$ is a linear function of $\cos \theta_{\mathrm{adv}}$. That is to say when $K_{\mathrm{a}}^{\prime}=K_{\mathrm{r}}^{\prime}=0$, the $\cos \theta_{\text {rec }} / \cos \theta_{\text {adv }}$ relationship can be reduced as a linear function.

The comparison with experiments indicates that the model proposed in this work can adequately fit the experimental data, while the prediction of other metastable contact angle models shows obvious deviation from experiments. In most cases, the fitting results of $t_{1 a}, t_{1 \mathrm{r}}, t_{2 a}$ and $t_{2 \mathrm{r}}$ satisfy the constraint of $\left|t_{1}\right|+\left|t_{2}\right|$ $\leq 1-\phi_{\mathrm{S}}$.

\section{CONCLUSIONS}

A contact angle hysteresis model is proposed in this work, which presumes a variable wetting state to explain the continuous change of the contact angle or multiple metastable angle phenomena during hysteresis. The presumptions combine the characteristics in the Wenzel model, Cassie-Baxter model, the impregnating model, and the mixed-state model. ${ }^{13,14,16,19}$ Unlike these models, the fraction of liquid or gas prefilling the grooves is assumed to be variable, so is the fraction of liquid or gas displaced by the other phase. The variable $t_{1}$ and $t_{2}$ may vary according to the external energy input, which is the external cause of change in surface energy. These two variables enable the continuous change of the contact angle and ensure a minimal surface energy during hysteresis. The advancing angle $\theta_{\text {adv }}$ and receding angles $\theta_{\text {rec }}$ are explicitly expressed by functions of the Young contact angle $\theta_{\mathrm{o}}$, surface topographical characteristics $\phi_{\mathrm{S}}$ and $r$, and variables $t_{1}$ and $t_{2}$. Our model is in good coincidence with experiments from the literature. ${ }^{4,12,23,26,29-33}$ The relationship between $\theta_{\text {rec }}$ and $\theta_{\text {adv }}$ can be further obtained through our model, which is able to fit the nonlinear or linear relationship between $\cos \theta_{\text {rec }}$ and $\cos \theta_{\text {adv }}$. Through fitting results, the computed height of a defect is found to be of the same magnitude with the particle decorating the solid surface, in the experiments of Ramos et al. ${ }^{12}$ This indicates that the fitted parameters $t_{1}$ and $t_{2}$ are acceptable. Furthermore, the estimation of $t_{1}$ and $t_{2}$ can be further developed to provide more accurate predictions.

\section{ASSOCIATED CONTENT}

\section{(S Supporting Information}

The Supporting Information is available free of charge on the ACS Publications website at DOI: 10.1021/acs.jpcc.9b03288.

Complete fitting results of model parameters; distribution of $t_{1} / t_{2}$ for unknown $\phi_{\mathrm{S}} / r$ experiments (PDF)

\section{AUTHOR INFORMATION}

\section{Corresponding Authors}

*E-mail: yunli@mail.xjtu.edu.cn (Y.L.).

*E-mail: linmian@imech.ac.cn (M.L.).

\section{ORCID $\odot$}

Yun Li: 0000-0003-4168-6400

\section{Notes}

The authors declare no competing financial interest.

\section{ACKNOWLEDGMENTS}

This work was supported by the National Natural Science Foundation of China (Grant No. 41690132), the Strategic Priority Research Program of the Chinese Academy of Sciences (Grant No. XDA14010304), the National Natural Science Foundation of China (Grant No. 41574129) and the Major National Science and Technology Special Program of China (Grant No. 2017ZX05037-001).

\section{REFERENCES}

(1) Young, T. An Essay on the Cohesion of Fluids. Philos. Trans. R. Soc. London 1805, 95, 65-87.

(2) Ramiasa, M.; Ralston, J.; Fetzer, R.; Sedev, R. The Influence of Topography on Dynamic Wetting. Adv. Colloid Interface Sci. 2014, 206, 275-293.

(3) Bonn, D.; Eggers, J.; Indekeu, J.; Meunier, J.; Rolley, E. Wetting and Spreading. Rev. Mod. Phys. 2009, 81, 739-805.

(4) Lei, D.; Lin, M.; Li, Y.; Jiang, W. A Two-Angle Model of Dynamic Wetting in Microscale Capillaries under Low Capillary Numbers with Experiments. J. Colloid Interface Sci. 2018, 520, 91-100.

(5) Quéré, D. Wetting and Roughness. Annu. Rev. Mater. Res. 2008, $38,71-99$.

(6) Bush, J. W. M.; Hu, D. L.; Prakash, M. The Integument of WaterWalking Arthropods: Form and Function. In Advances in Insect Physiology; Casas, J., Simpson, S. J., Eds.; Academic Press, 2007; Vol. 34, pp 117-192.

(7) Jamin, M. J. Xxvii. On the Equilibrium and Motion of Liquids in Porous Bodies. London, Edinburgh Dublin Philos. Mag. J. Sci. 1860, 19, 204-207.

(8) Smith, W. O.; Crane, M. D. The Jamin Effect in Cylindrical Tubes. J. Am. Chem. Soc. 1930, 52, 1345-1349.

(9) Morrow, N. R.; Nguyen, M. D. Effect of Interface Velocity on Dynamic Contact Angles at Rough Surfaces. J. Colloid Interface Sci. 1982, 89, 523-531.

(10) Meiron, T. S.; Marmur, A.; Saguy, I. S. Contact Angle Measurement on Rough Surfaces. J. Colloid Interface Sci. 2004, 274, 637-44.

(11) Ramos, S. M.; Charlaix, E.; Benyagoub, A.; Toulemonde, M. Wetting on Nanorough Surfaces. Phys. Rev. E 2003, 67, No. 031604.

(12) Ramos, S.; Tanguy, A. Pinning-Depinning of the Contact Line on Nanorough Surfaces. Eur. Phys. J. E 2006, 19, 433-440.

(13) Wenzel, R. N. Resistance of Solid Surfaces to Wetting by Water. Ind. Eng. Chem. 1936, 28, 988-994.

(14) Cassie, A. B. D.; Baxter, S. Wettability of Porous Surfaces. Trans. Faraday Soc. 1944, 40, 546-551.

(15) Wolansky, G.; Marmur, A. Apparent Contact Angles on Rough Surfaces: The Wenzel Equation Revisited. Colloids Surf., A 1999, 156, 381-388.

(16) Bico, J.; Thiele, U.; Quéré, D. Wetting of Textured Surfaces. Colloids Surf., A 2002, 206, 41-46.

(17) Reyssat, M.; Quéré, D. Contact Angle Hysteresis Generated by Strong Dilute Defects. J. Phys. Chem. B 2009, 113, 3906-3909.

(18) Bormashenko, E. Progress in Understanding Wetting Transitions on Rough Surfaces. Adv. Colloid Interface Sci. 2015, 222, 92-103.

(19) Miwa, M.; Nakajima, A.; Fujishima, A.; Hashimoto, K.; Watanabe, T. Effects of the Surface Roughness on Sliding Angles of Water Droplets on Superhydrophobic Surfaces. Langmuir 2000, 16, 5754-5760.

(20) Marmur, A. Contact Angle Hysteresis on Heterogeneous Smooth Surfaces. J. Colloid Interface Sci. 1994, 168, 40-46.

(21) Marmur, A. Wetting on Hydrophobic Rough Surfaces: To Be Heterogeneous or Not to Be? Langmuir 2003, 19, 8343-8348.

(22) Joanny, J. F.; de Gennes, P. G. A Model for Contact Angle Hysteresis. J. Phys. Chem. A 1984, 81, 552-562. 
(23) Koch, B. M. L.; Amirfazli, A.; Elliott, J. A. W. Modeling and Measurement of Contact Angle Hysteresis on Textured High-ContactAngle Surfaces. J. Phys. Chem. C 2014, 118, 18554-18563.

(24) Lei, D.; Lin, M.; Li, Y.; Jiang, W. A Two-Angle Model of Dynamic Wetting in Microscale Capillaries under Low Capillary Numbers with Experiments. J. Colloid Interface Sci. 2018, 520, 91-100.

(25) Tartakovsky, A. M.; Trask, N.; Pan, K.; Jones, B.; Pan, W.; Williams, J. R. Smoothed Particle Hydrodynamics and Its Applications for Multiphase Flow and Reactive Transport in Porous Media. Comput. Geosci. 2016, 20, 807-834.

(26) Yeong, Y. H.; Milionis, A.; Loth, E.; Bayer, I. S. Microscopic Receding Contact Line Dynamics on Pillar and Irregular Superhydrophobic Surfaces. Sci. Rep. 2015, 5, No. 8384.

(27) Marmur, A.; Della Volpe, C.; Siboni, S.; Amirfazli, A.; Drelich, J. Contact Angles and Wettability: Towards Common and Accurate Terminology. Surf. Innovations 2017, 5, 1-24.

(28) Priest, C.; Albrecht, T. W. J.; Sedev, R.; Ralston, J. Asymmetric Wetting Hysteresis on Hydrophobic Microstructured Surfaces. Langmuir 2009, 25, 5655-5660.

(29) Priest, C.; Sedev, R.; Ralston, J. A Quantitative Experimental Study of Wetting Hysteresis on Discrete and Continuous Chemical Heterogeneities. Colloid Polym. Sci. 2013, 291, 271-277.

(30) Lam, C. N. C.; Wu, R.; Li, D.; Hair, M. L.; Neumann, A. W. Study of the Advancing and Receding Contact Angles: Liquid Sorption as a Cause of Contact Angle Hysteresis. Adv. Colloid Interface Sci. 2002, 96, 169-191.

(31) Johnson, R. E.; Dettre, R. H.; Brandreth, D. A. Dynamic Contact Angles and Contact Angle Hysteresis. J. Colloid Interface Sci. 1977, 62, 205-212.

(32) Schneemilch, M.; Hayes, R. A.; Petrov, J. G.; Ralston, J. Dynamic Wetting and Dewetting of a Low-Energy Surface by Pure Liquids. Langmuir 1998, 14, 7047-7051.

(33) Petrov, J. G.; Ralston, J.; Schneemilch, M.; Hayes, R. A. Dynamics of Partial Wetting and Dewetting in Well-Defined Systems. J. Phys. Chem. B 2003, 107, 1634-1645. 